\title{
Three-Component Synthesis of 2-Oxoindolin-3-ylphosphonates
}

\author{
Ghazaleh Imani Shakibaei, ${ }^{a}$ Afsaneh Feiz, ${ }^{a}$ Ramin Ghahremanzadeh, ${ }^{b}$ and Ayoob Bazgir ${ }^{*}, a$ \\ ${ }^{a}$ Department of Chemistry, Shahid Beheshti University; General Campus, Tehran 1983963113, Iran: and \\ ${ }^{b}$ Nanobiotechnology Research Center, Avicenna Research Institute; ACECR, Tehran 1936773493, Iran. \\ Received January 24, 2010; accepted April 12, 2010; published online April 20, 2010
}

\begin{abstract}
A one-pot, three-component and catalyst-free method for the efficient and simple synthesis of dialkyl 3-(dicyanomethyl)-2-oxoindolin-3-ylphosphonates at $50^{\circ} \mathrm{C}$ under solvent-free conditions is reported. The features of this procedure are mild reaction conditions, high yields of products, and operational simplicity.
\end{abstract}

Key words isatin; oxindole; dialkyl phosphite; oxoindolin-3-ylphosphonate; acenaphthylen-1,2-dione

Multicomponent reactions (MCRs), in which multiple reactions are combined into the synthetic operation have been used extensively to form carbon-carbon bonds in the synthetic chemistry. ${ }^{1-4)}$ Such reactions offer a wide range of possibilities for the efficient construction of highly complex molecules in a single procedural step, thus avoiding the complicated purification operations and allowing savings of both solvents and reagents. In the past decade, there has been tremendous development in three- and four-component reactions, and great efforts continue to be made to develop new MCRs. ${ }^{5-9)}$ In this context, oxindole derivatives show interesting features that make them attractive for use in MCRs. Oxindoles are known to possess antibacterial, antiprotozoal, and anti-inflammatory activities and are also patented as PR (progesterone receptors) agonists. ${ }^{10-14)}$ The naturally occurring oxindole derivative convolutamydine has been found to exhibit potent activity in the differentiation of HL-60 human plomyelocytic leukemic cells. ${ }^{15)}$ The varied biological activities of oxindole derivatives have attracted the synthetic chemists to a number of synthetic strategies. ${ }^{16-20)}$

Pioneering work on $\mathrm{P}-\mathrm{C}$ bond formation was carried out by Arbusov in the early 20th century, culminating in the well-known Michaelis-Arbusov reaction. ${ }^{21)}$ In the following decades the chemistry of phosphonates developed relatively slowly because of the difficulty in formation of the $\mathrm{C}-\mathrm{P}$ bond. Its renaissance came after 1959 with the discovery of naturally occurring aminophosphonic acids ${ }^{22}$ and new biologically active phosphonates. ${ }^{23)}$ Organophosphorus compounds have found a wide range of application in the areas of industrial, agricultural, and medicinal chemistry owing to their biological and physical properties as well as their utility as synthetic intermediates. ${ }^{24-26)} \alpha$-Functionalized phosphonic acids are valuable intermediates for the preparation of medicinal compounds and synthetic intermediates. ${ }^{27-29)}$ Natural products containing $\mathrm{P}-\mathrm{C}$ bonds also show interesting biological activities. ${ }^{30,31)}$ Among various methods to generate $\mathrm{P}-\mathrm{C}$ bonds, the addition of $\mathrm{P}(\mathrm{O})-\mathrm{H}$ bonds across alkenes is one of the most utilized. ${ }^{32,33)}$ There are three general approaches: (a) the phospha-Michael reaction of activated alkenes, most commonly promoted by catalyst ${ }^{32-37)}$ or microwave $^{38)}$; (b) addition to inactivated olefins promoted by radical initiators ${ }^{39}$; (c) hydrophosphorylation of inactivated alkenes catalyzed by transition metals. ${ }^{40,41)}$ Recently, threecomponent methods have been reported for the synthesis of organophosphorus derivatives. ${ }^{42,43)}$

Considering the above reports, and as a continue of our

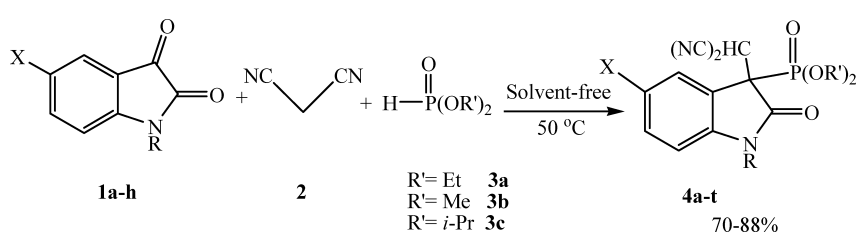

Chart 1. Synthesis of 2-Oxoindolin-3-ylphosphonates 4

work on the synthesis of heterocyclic compounds, ${ }^{44-58)}$ we are currently investigating the synthesis of various 2-oxoindolin-3-ylphosphonates via a facile, atom-economical, and one-pot, three-component condensation reaction of isatins $\mathbf{1}$, malononitril $\mathbf{2}$ and dialkyl phosphites $\mathbf{3}$ under solvent-free conditions at $50{ }^{\circ} \mathrm{C}$ (Chart 1$)$.

\section{Results and Discussion}

In a pilot experiment, the reaction of isatin 1a, malononitril $\mathbf{2}$ and diethyl phosphite $\mathbf{3 a}$ in the absence of any catalyst proceeds under solvent-free conditions at $50^{\circ} \mathrm{C}$. The progress of the reaction was monitored by TLC. After completion of the reaction after $2 \mathrm{~h}$, the product diethyl 3-(dicyanomethyl)-2-oxoindolin-3-ylphosphonate $\mathbf{4 a}$ was obtained in $81 \%$ yield.

Then, to delineate this approach, particularly in regard to library construction, this methodology was evaluated by using eight substituted isatins $\mathbf{1 a - h}$, three dialkyl phosphates $\mathbf{3} \mathbf{a}-\mathbf{c}$ and malononitril $\mathbf{2}$ and corresponding dialkyl 3-(dicyanomethyl)-2-oxoindolin-3-ylphosphonates $4 \mathbf{a}-\mathbf{t}$ were selectively synthesized by the one-pot, three-component condensation reaction in good yields under similar conditions for $1-8 \mathrm{~h}$ (Table 1$)$.

To the best of our knowledge, this new procedure provides the first example of a three-component and catalyst-free synthesis of dialkyl 3-(dicyanomethyl)-2-oxoindolin-3-ylphosphonates. The reactions under catalyst- and solvent-free conditions are considerably safe, nontoxic, environmentally friendly, and inexpensive. The absence of catalyst for the reaction allows avoiding the use of moisture sensitive and heavy metal Lewis acids. This method, based on catalyst-free reaction under solvent-free conditions, is the most simple and convenient and would be applicable for the synthesis of different types of dicyanomethyl-oxoindolin-3-ylphosphonates.

${ }^{1} \mathrm{H}$ - and ${ }^{13} \mathrm{C}-\mathrm{NMR}$ spectra of the crude products clearly indicated the formation of 2-oxoindolin-3-ylphosphonates 4 . Compounds 4 are stable solids whose structures were estab- 
Table 1. 2-Oxoindolin-3-ylphosphonates 4

\begin{tabular}{|c|c|c|c|c|c|}
\hline Compound 4 & $\mathrm{X}$ & $\mathrm{R}$ & $\mathrm{R}^{\prime}$ & Time (h) & Yield $(\%)^{a)}$ \\
\hline $\mathbf{a}$ & $\mathrm{H}$ & $\mathrm{H}$ & $\mathrm{Et}$ & 2 & 81 \\
\hline b & $\mathrm{H}$ & $\mathrm{PhCH}_{2}$ & Et & 3 & 82 \\
\hline c & $\mathrm{H}$ & $\mathrm{Me}$ & $\mathrm{Et}$ & 5 & 85 \\
\hline d & $\mathrm{NO}_{2}$ & $\mathrm{H}$ & $\mathrm{Et}$ & 2 & 70 \\
\hline $\mathbf{e}$ & $\mathrm{Br}$ & $\mathrm{H}$ & Et & 1.5 & 83 \\
\hline f & $\mathrm{H}$ & $\mathrm{H}$ & $\mathrm{Me}$ & 2 & 71 \\
\hline $\mathbf{g}$ & $\mathrm{H}$ & $\mathrm{PhCH}_{2}$ & $\mathrm{Me}$ & 1 & 75 \\
\hline h & $\mathrm{H}$ & $\mathrm{Me}$ & $\mathrm{Me}$ & 3 & 70 \\
\hline $\mathbf{i}$ & $\mathrm{H}$ & Et & $\mathrm{Me}$ & 3 & 86 \\
\hline $\mathbf{j}$ & $\mathrm{NO}_{2}$ & $\mathrm{H}$ & $\mathrm{Me}$ & 2 & 72 \\
\hline $\mathbf{k}$ & $\mathrm{Br}$ & $\mathrm{H}$ & $\mathrm{Me}$ & 2 & 88 \\
\hline 1 & $\mathrm{Br}$ & $\mathrm{Me}$ & $\mathrm{Me}$ & 4 & 82 \\
\hline $\mathbf{m}$ & $\mathrm{H}$ & $\mathrm{H}$ & $i$-Pr & 8 & 81 \\
\hline $\mathbf{n}$ & $\mathrm{H}$ & $\mathrm{PhCH}_{2}$ & $i$-Pr & 3 & 76 \\
\hline o & $\mathrm{H}$ & $\mathrm{Me}$ & $i$-Pr & 7 & 72 \\
\hline p & $\mathrm{H}$ & Et & $i$-Pr & 8 & 74 \\
\hline $\mathbf{q}$ & $\mathrm{NO}_{2}$ & $\mathrm{H}$ & $i$-Pr & 3 & 71 \\
\hline $\mathbf{r}$ & $\mathrm{Br}$ & $\mathrm{H}$ & $i$-Pr & 2 & 79 \\
\hline $\mathbf{s}$ & $\mathrm{Br}$ & $\mathrm{Me}$ & $i$-Pr & 6 & 80 \\
\hline $\mathbf{t}$ & $\mathrm{NO}_{2}$ & $\mathrm{Me}$ & $i$-Pr & 6 & 81 \\
\hline
\end{tabular}

a) Isolated yields.

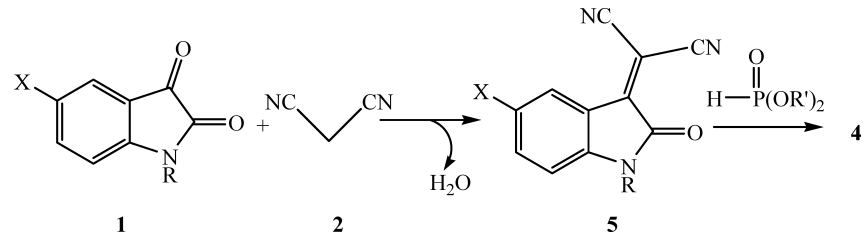

Chart 2. Possible Mechanism for the Formation of Products 4

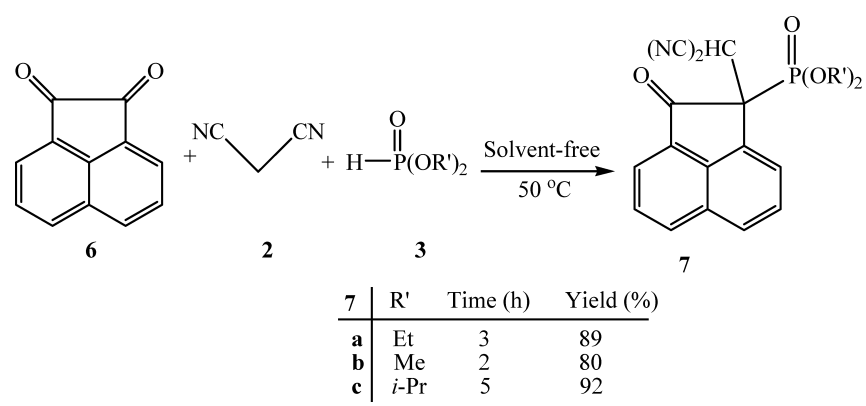

Chart 3. Synthesis of 2-Oxo-1,2-dihydroacenaphthylen-1-ylphosphonates

lished by IR, ${ }^{1} \mathrm{H}-,{ }^{31} \mathrm{P}$ - and ${ }^{13} \mathrm{C}-\mathrm{NMR}$ spectroscopy and elemental analysis.

The formation of products 4 can be rationalized by initial formation of 2-(2-oxoindolin-3-ylidene)malononitriles $\mathbf{5}$ by standard Knoevenagel condensation of isatins $\mathbf{1}$ and malononitrile 2. Subsequent Michael-type addition of dialkyl phosphates $\mathbf{3}$ to the intermediate $\mathbf{5}$ afforded the corresponding products 4 (Chart 2).

As expected, when the isatin 1 was replaced by acenaphthylene-1,2-dione 6, dialkyl 1-(dicyanomethyl)-2-oxo-1,2-dihydroacenaphthylen-1-ylphosphonates $\mathbf{7 a - c}$ was obtained in good yield under the same reaction conditions (Chart 3). These reactions proceeded very cleanly under mild conditions, and no undesirable side reactions were observed. The acenaphthylen-1-ylphosphonates $\mathbf{7 a}$ - c were synthesized for the first time.

\section{Conclusion}

In conclusion, an efficient, atom-economical, and simple method for the preparation of dialkyl 3-(dicyanomethyl)-2oxoindolin-3-ylphosphonate and dialkyl 1-(dicyanomethyl)2-oxo-1,2-dihydroacenaphthylen-1-ylphosphonates using readily available starting materials under solvent-free conditions is reported. Prominent among the advantages of this new catalyst-free method are operational simplicity, good yields and easy work-up procedures employed.

\section{Experimental}

General Melting points were measured on an Electrothermal 9100 apparatus and are uncorrected. IR spectra were recorded on a Shimadzu IR470 spectrometer. ${ }^{1} \mathrm{H}$ - and ${ }^{13} \mathrm{C}-\mathrm{NMR}$ spectra were recorded on a BRUKER DRX-300 AVANCE spectrometer at 300.13 and $75.47 \mathrm{MHz}$, respectively. Elemental analyses were performed using a Heracus CHN-O-Rapid analyzer.

Typical Procedure for Preparation of Diethyl 3-(Dicyanomethyl)-2-oxoindolin-3-ylphosphonate 4a A mixture of isatin $(0.15 \mathrm{~g}, 1 \mathrm{mmol})$, malononitril $(0.66 \mathrm{~g}, 1 \mathrm{mmol})$ and diethyl phosphite $(0.16 \mathrm{~g}, 1.1 \mathrm{mmol})$ was stirred at $50^{\circ} \mathrm{C}$ for $2 \mathrm{~h}$. After completion of the reaction confirmed by TLC (eluent: EtOAc/ $n$-hexane, $1: 1$ ), the reaction mixture was washed with diethyl ether $(5 \mathrm{ml})$ and residue recrystallized from $\mathrm{MeOH}-\mathrm{H}_{2} \mathrm{O}(1: 3)$ to afford the pure product $4 \mathrm{a}$ as a cream powder $(0.27 \mathrm{~g}, 81 \%)$. mp $168-170^{\circ} \mathrm{C}$. IR $(\mathrm{KBr})\left(v_{\max } / \mathrm{cm}^{-1}\right): 3432,2249,1733,1240 .{ }^{1} \mathrm{H}-\mathrm{NMR}\left(\mathrm{DMSO}-d_{6}\right) \delta$ : $1.14\left(3 \mathrm{H}, \mathrm{t},{ }^{3} J_{\mathrm{HH}}=7.0 \mathrm{~Hz}, \mathrm{CH}_{3}\right), 1.25\left(3 \mathrm{H}, \mathrm{t},{ }^{3} J_{\mathrm{HH}}=7.0 \mathrm{~Hz}, \mathrm{CH}_{3}\right), 3.98-4.16$ $\left(4 \mathrm{H}, \mathrm{m}, \mathrm{CH}_{2}\right), 5.90\left(1 \mathrm{H}, \mathrm{d},{ }^{3} \mathrm{~J}_{\mathrm{HP}}=8.0 \mathrm{~Hz}, \mathrm{CH}(\mathrm{CN})_{2}\right), 7.00-7.57(4 \mathrm{H}, \mathrm{m}, \mathrm{H}-$ Ar), $11.38(1 \mathrm{H}, \mathrm{s}, \mathrm{NH}) \cdot{ }^{13} \mathrm{C}-\mathrm{NMR}\left(\mathrm{DMSO}-d_{6}\right) \delta: 16.3\left(\mathrm{~d},{ }^{3} J_{\mathrm{CP}}=5.7 \mathrm{~Hz}\right.$, $\left.\mathrm{CH}_{3}\right), 16.4\left(\mathrm{~d},{ }^{3} J_{\mathrm{CP}}=5.9 \mathrm{~Hz}, \mathrm{CH}_{3}\right), 27.5,53.1\left(\mathrm{~d},{ }^{1} J_{\mathrm{CP}}=137.8 \mathrm{~Hz}, \mathrm{C}-\mathrm{P}\right), 64.7$ $\left(\mathrm{d},{ }^{2} J_{\mathrm{CP}}=7.2 \mathrm{~Hz}, \mathrm{OCH}_{2}\right), 65.4\left(\mathrm{~d},{ }^{2} J_{\mathrm{CP}}=6.9 \mathrm{~Hz}, \mathrm{OCH}_{2}\right), 111.1,111.6(\mathrm{~d}$ $\left.{ }^{3} J_{\mathrm{CP}}=13.7 \mathrm{~Hz}, \mathrm{CN}\right), 112.0\left(\mathrm{~d},{ }^{3} J_{\mathrm{CP}}=7.6 \mathrm{~Hz}, \mathrm{CN}\right), 122.0\left(\mathrm{~d},{ }^{2} J_{\mathrm{CP}}=5.5 \mathrm{~Hz}, \mathrm{H}-\right.$ $\mathrm{Ar}), 123.1,126.2,131.4,143.4\left(\mathrm{~d},{ }^{3} J_{\mathrm{CP}}=6.8 \mathrm{~Hz}, \mathrm{H}-\mathrm{Ar}\right), 171.0(\mathrm{~d}$ $\left.{ }^{2} J_{\mathrm{CP}}=3.1 \mathrm{~Hz}, \mathrm{CO}\right) .{ }^{31} \mathrm{P}-\mathrm{NMR}$ (DMSO- $\left.d_{6}\right) \delta$ : 13.01. Anal. Calcd for $\mathrm{C}_{15} \mathrm{H}_{16} \mathrm{~N}_{3} \mathrm{O}_{4} \mathrm{P}: \mathrm{C}, 54.06 ; \mathrm{H}, 4.84 ; \mathrm{N}, 12.61 \%$. Found: $\mathrm{C}, 54.15 ; \mathrm{H}, 4.78 ; \mathrm{N}$, $12.53 \%$

Diethyl 1-Benzyl-3-(dicyanomethyl)-2-oxoindolin-3-ylphosphonate 4b $82 \%$ yield. Pink powder. mp $177-180^{\circ} \mathrm{C}$. IR $(\mathrm{KBr})\left(v_{\max } / \mathrm{cm}^{-1}\right): 2260$, 1720, 1229. ${ }^{1} \mathrm{H}-\mathrm{NMR}$ (DMSO- $\left.d_{6}\right) \delta: 1.07\left(3 \mathrm{H}, \mathrm{t}, J_{\mathrm{HH}}=6.9 \mathrm{~Hz}, \mathrm{CH}_{3}\right), 1.24$ $\left(3 \mathrm{H}, \mathrm{t}, J_{\mathrm{HH}}=6.9 \mathrm{~Hz}, \mathrm{CH}_{3}\right), 3.89-4.14\left(4 \mathrm{H}, \mathrm{m}, \mathrm{CH}_{2}\right), 4.97,5.06\left(2 \mathrm{H}, \mathrm{AB}_{\mathrm{g}}\right.$, $\left.J_{\mathrm{AB}}=15.9 \mathrm{~Hz}, \mathrm{NCH}_{2}\right), 6.06\left(1 \mathrm{H}, \mathrm{d}, J_{\mathrm{HP}}=8.1 \mathrm{~Hz}, \mathrm{CH}(\mathrm{CN})_{2}\right), 6.06-7.63(9 \mathrm{H}$, m, H-Ar). ${ }^{13} \mathrm{C}-\mathrm{NMR}$ (DMSO- $\left.d_{6}\right) \delta: 16.3\left(\mathrm{~d}, J_{\mathrm{CP}}=5.5 \mathrm{~Hz}, \mathrm{CH}_{3}\right), 16.4(\mathrm{~d}$, $\left.J_{\mathrm{CP}}=5.6 \mathrm{~Hz}, \mathrm{CH}_{3}\right), 27.4,43.9,52.6\left(\mathrm{~d}, J_{\mathrm{CP}}=137.4 \mathrm{~Hz}, \mathrm{C}-\mathrm{P}\right), 65.0(\mathrm{~d}$, $\left.J_{\mathrm{CP}}=7.6 \mathrm{~Hz}, \quad \mathrm{OCH}_{2}\right), 65.6\left(\mathrm{~d}, J_{\mathrm{CP}}=6.8 \mathrm{~Hz}, \mathrm{OCH}_{2}\right), 110.9,111.6(\mathrm{~d}$, $\left.J_{\mathrm{CP}}=12.7 \mathrm{~Hz}, \mathrm{CN}\right), 111.9\left(\mathrm{~d}, J_{\mathrm{CP}}=7.9 \mathrm{~Hz}, \mathrm{CN}\right), 121.2\left(\mathrm{~d}, J_{\mathrm{CP}}=5.9 \mathrm{~Hz}, \mathrm{C}-\mathrm{Ar}\right)$, $123.9,126.1\left(\mathrm{~d}, J_{\mathrm{CP}}=2.9 \mathrm{~Hz}, \mathrm{C}-\mathrm{Ar}\right), 127.7,128.1,129.0,131.5,135.6,143.7$ $\left(\mathrm{d}, J_{\mathrm{CP}}=6.5 \mathrm{~Hz}, \mathrm{C}-\mathrm{Ar}\right), 169.6\left(\mathrm{~d}, J_{\mathrm{CP}}=3.5 \mathrm{~Hz}, \mathrm{CO}\right) .{ }^{31} \mathrm{P}-\mathrm{NMR}\left(\mathrm{DMSO}-d_{6}\right) \delta$ : 13.02. Anal. Calcd for $\mathrm{C}_{22} \mathrm{H}_{22} \mathrm{~N}_{3} \mathrm{O}_{4} \mathrm{P}: \mathrm{C}, 62.41 ; \mathrm{H}, 5.24 ; \mathrm{N}, 9.92 \%$. Found: $\mathrm{C}$, 62.33 ; H, 5.29; N, 9.85\%.

Diethyl 3-(Dicyanomethyl)-1-methyl-2-oxoindolin-3-ylphosphonate 4c $85 \%$ yield. White powder. $\mathrm{mp} 100-104^{\circ} \mathrm{C}$. IR $(\mathrm{KBr})\left(v_{\max } / \mathrm{cm}^{-1}\right): 2260$ 1713, 1256. ${ }^{1} \mathrm{H}-\mathrm{NMR}\left(\mathrm{DMSO}-d_{6}\right) \delta: 1.16\left(3 \mathrm{H}, \mathrm{t}, J_{\mathrm{HH}}=6.9 \mathrm{~Hz}, \mathrm{CH}_{3}\right), 1.24$ $\left(3 \mathrm{H}, \mathrm{t}, J_{\mathrm{HH}}=6.9 \mathrm{~Hz}, \mathrm{CH}_{3}\right), 3.23\left(3 \mathrm{H}, \mathrm{s}, \mathrm{CH}_{3}\right), 3.96-4.15\left(4 \mathrm{H}, \mathrm{m}, \mathrm{CH}_{2}\right), 5.95$ $\left(1 \mathrm{H}, \mathrm{d}, J_{\mathrm{HP}}=8.4 \mathrm{~Hz}, \mathrm{CH}(\mathrm{CN})_{2}\right), 7.22-7.61(4 \mathrm{H}, \mathrm{m}, \mathrm{H}-\mathrm{Ar}) .{ }^{13} \mathrm{C}-\mathrm{NMR}$ $\left(\mathrm{DMSO}-d_{6}\right) \delta: 16.3\left(\mathrm{~d}, J_{\mathrm{CP}}=5.7 \mathrm{~Hz}, \mathrm{CH}_{3}\right), 16.4\left(\mathrm{~d}, J_{\mathrm{CP}}=5.8 \mathrm{~Hz}, \mathrm{CH}_{3}\right), 27.4$, $27.5,52.6\left(\mathrm{~d}, J_{\mathrm{CP}}=137.8 \mathrm{~Hz}, \mathrm{C}-\mathrm{P}\right), 64.8\left(\mathrm{~d}, J_{\mathrm{CP}}=7.4 \mathrm{~Hz}, \mathrm{OCH}_{2}\right), 65.5(\mathrm{~d}$, $\left.J_{\mathrm{CP}}=8.1 \mathrm{~Hz}, \quad \mathrm{OCH}_{2}\right), 110.3,111.5 \quad\left(\mathrm{~d}, J_{\mathrm{CP}}=12.4 \mathrm{~Hz}, \mathrm{CN}\right), 111.9 \quad(\mathrm{~d}$, $\left.J_{\mathrm{CP}}=7.6 \mathrm{~Hz}, \mathrm{CN}\right), 121.2\left(\mathrm{~d}, J_{\mathrm{CP}}=5.5 \mathrm{~Hz}, \mathrm{C}-\mathrm{Ar}\right), 123.7,125.9,131.5,144.7$ $\left(\mathrm{d}, J_{\mathrm{CP}}=6.5 \mathrm{~Hz}, \mathrm{C}-\mathrm{Ar}\right), 169.4 .{ }^{31} \mathrm{P}-\mathrm{NMR}\left(\mathrm{DMSO}-d_{6}\right) \delta: 12.75$. Anal. Calcd for $\mathrm{C}_{16} \mathrm{H}_{18} \mathrm{~N}_{3} \mathrm{O}_{4} \mathrm{P}: \mathrm{C}, 55.33 ; \mathrm{H}, 5.22 ; \mathrm{N}, 12.10 \%$. Found: $\mathrm{C}, 55.27 ; \mathrm{H}, 5.15$; $\mathrm{N}, 12.17 \%$.

Diethyl 3-(Dicyanomethyl)-5-nitro-2-oxoindolin-3-ylphosphonate 4d $70 \%$ yield. Yellow powder. $\mathrm{mp} 185-190{ }^{\circ} \mathrm{C}$. IR $(\mathrm{KBr})\left(v_{\max } / \mathrm{cm}^{-1}\right): 3458$, 2260, 1742, 1250. ${ }^{1} \mathrm{H}-\mathrm{NMR}$ (DMSO- $\left.d_{6}\right) \delta: 1.17\left(3 \mathrm{H}, \mathrm{t}, J_{\mathrm{HH}}=6.9 \mathrm{~Hz}, \mathrm{CH}_{3}\right)$, $1.27\left(3 \mathrm{H}, \mathrm{t}, J_{\mathrm{HH}}=6.9 \mathrm{~Hz} \mathrm{CH} \mathrm{CH}_{3}\right), 4.04-4.21\left(4 \mathrm{H}, \mathrm{m}, \mathrm{CH}_{2}\right), 6.17(1 \mathrm{H}, \mathrm{d}$, $\left.J_{\mathrm{HP}}=8.7 \mathrm{~Hz}, \mathrm{CH}(\mathrm{CN})_{2}\right), 7.23-8.40(3 \mathrm{H}, \mathrm{m}, \mathrm{H}-\mathrm{Ar}), 12.13(1 \mathrm{H}, \mathrm{s}, \mathrm{NH}) .{ }^{13} \mathrm{C}-$ NMR (DMSO- $\left.d_{6}\right) \delta: 16.3\left(\mathrm{~d}, J_{\mathrm{CP}}=2.0 \mathrm{~Hz}, \mathrm{CH}_{3}\right), 16.4\left(\mathrm{~d}, J_{\mathrm{CP}}=2.2 \mathrm{~Hz}, \mathrm{CH}_{3}\right)$, 26.8, 27.5, $52.8\left(\mathrm{~d}, J_{\mathrm{CP}}=136.3 \mathrm{~Hz}, \mathrm{C}-\mathrm{P}\right), 65.3\left(\mathrm{~d}, J_{\mathrm{CP}}=7.5 \mathrm{~Hz}, \mathrm{OCH}_{2}\right), 66.1$ $\left(\mathrm{d}, J_{\mathrm{CP}}=6.9 \mathrm{~Hz}, \mathrm{OCH}_{2}\right), 111.1,111.6,121.9\left(\mathrm{~d}, J_{\mathrm{CP}}=3.1 \mathrm{~Hz}, \mathrm{C}-\mathrm{Ar}\right), 123.0$, $128.5,143.0\left(\mathrm{~d}, J_{\mathrm{CP}}=3.0 \mathrm{~Hz}, \mathrm{C}-\mathrm{Ar}\right), 149.5,149.6,171.4 .{ }^{31} \mathrm{P}-\mathrm{NMR}$ (DMSO- 
$\left.d_{6}\right) \delta$ : 11.60. Anal. Calcd for $\mathrm{C}_{15} \mathrm{H}_{15} \mathrm{~N}_{4} \mathrm{O}_{6} \mathrm{P}: \mathrm{C}, 47.63 ; \mathrm{H}, 4.00 ; \mathrm{N}, 14.81 \%$. Found: C, 47.70; H, 3.92; N, 14.72\%.

Diethyl 5-Bromo-3-(dicyanomethyl)-2-oxoindolin-3-ylphosphonate 4e $83 \%$ yield. White powder. mp $182-185^{\circ} \mathrm{C}$. IR $(\mathrm{KBr})\left(v_{\max } / \mathrm{cm}^{-1}\right): 3421$, 2254, 1723, 1245. ${ }^{1} \mathrm{H}-\mathrm{NMR}\left(\mathrm{DMSO}-d_{6}\right) \delta: 1.15\left(3 \mathrm{H}, \mathrm{t}, J_{\mathrm{HH}}=6.9 \mathrm{~Hz}, \mathrm{CH}_{3}\right)$, $1.26\left(3 \mathrm{H}, \mathrm{t}, J_{\mathrm{HH}}=6.9 \mathrm{~Hz} \mathrm{CH}_{3}\right), 3.98-4.19\left(4 \mathrm{H}, \mathrm{m}, \mathrm{CH}_{2}\right), 5.99(1 \mathrm{H}, \mathrm{d}$, $\left.J_{\mathrm{HP}}=8.4 \mathrm{~Hz}, \mathrm{CH}(\mathrm{CN})_{2}\right), 6.98-7.63(3 \mathrm{H}, \mathrm{m}, \mathrm{H}-\mathrm{Ar}), 11.54(1 \mathrm{H}, \mathrm{s}, \mathrm{NH}) .{ }^{13} \mathrm{C}-$ NMR (DMSO- $\left.d_{6}\right) \delta: 16.3\left(\mathrm{~d}, J_{\mathrm{CP}}=5.1 \mathrm{~Hz}, \mathrm{CH}_{3}\right), 16.4\left(\mathrm{~d}, J_{\mathrm{CP}}=5.2 \mathrm{~Hz}, \mathrm{CH}_{3}\right)$, $27.1,52.9\left(\mathrm{~d}, J_{\mathrm{CP}}=137.1 \mathrm{~Hz}, \mathrm{C}-\mathrm{P}\right), 65.0\left(\mathrm{~d}, J_{\mathrm{CP}}=7.4 \mathrm{~Hz}, \mathrm{OCH}_{2}\right), 65.7(\mathrm{~d}$, $\left.J_{\mathrm{CP}}=7.0 \mathrm{~Hz}, \mathrm{OCH}_{2}\right), 111.3,111.8\left(\mathrm{~d}, J_{\mathrm{CP}}=8.4 \mathrm{~Hz}, \mathrm{CN}\right), 113.1,114.3,124.3$, $128.8,134.2,142.8$ (d, $\left.J_{\mathrm{CP}}=6.9 \mathrm{~Hz}, \mathrm{C}-\mathrm{Ar}\right), 170.5 .{ }^{31} \mathrm{P}-\mathrm{NMR}\left(\mathrm{DMSO}-d_{6}\right) \delta$ 12.30. Anal. Calcd for $\mathrm{C}_{15} \mathrm{H}_{15} \mathrm{BrN}_{3} \mathrm{O}_{4} \mathrm{P}: \mathrm{C}, 43.71 ; \mathrm{H}, 3.67 ; \mathrm{N}, 10.19 \%$. Found: C, 43.64; H, 3.60; N, 10.11\%.

Dimethyl 3-(Dicyanomethyl)-2-oxoindolin-3-ylphosphonate 4f 71\% yield. White powder. mp $192-194^{\circ} \mathrm{C}$. IR (KBr) $\left(v_{\max } / \mathrm{cm}^{-1}\right): 3416,2260$, $1717,1254 .{ }^{1} \mathrm{H}-\mathrm{NMR}\left(\mathrm{DMSO}-d_{6}\right) \delta: 3.57\left(3 \mathrm{H}, \mathrm{d}, J_{\mathrm{HP}}=11.1 \mathrm{~Hz}, \mathrm{OCH}_{3}\right), 3.75$ $\left(3 \mathrm{H}, \mathrm{d}, J_{\mathrm{HP}}=11.1 \mathrm{~Hz}, \mathrm{OCH}_{3}\right), 5.96\left(1 \mathrm{H}, \mathrm{d}, J_{\mathrm{HP}}=8.1 \mathrm{~Hz}, \mathrm{CH}(\mathrm{CN})_{2}\right), 7.00$ 7.54 (4H, m, H-Ar), $11.38(1 \mathrm{H}, \mathrm{s}, \mathrm{NH}) .{ }^{13} \mathrm{C}-\mathrm{NMR}$ (DMSO-d $) \delta: 27.4,53.2$ $\left(\mathrm{d}, J_{\mathrm{CP}}=138.9 \mathrm{~Hz}, \mathrm{C}-\mathrm{P}\right), 55.0\left(\mathrm{~d}, J_{\mathrm{CP}}=7.5 \mathrm{~Hz}, \mathrm{OCH}_{3}\right), 55.8\left(\mathrm{~d}, J_{\mathrm{CP}}=6.7 \mathrm{~Hz}\right.$, $\left.\mathrm{OCH}_{3}\right), 111.2,111.8,121.8,123.1,126.1,131.5,143.3,143.4,170.8 .{ }^{31} \mathrm{P}$ NMR (DMSO- $\left.d_{6}\right) \delta$ : 15.42. Anal. Calcd for $\mathrm{C}_{13} \mathrm{H}_{12} \mathrm{~N}_{3} \mathrm{O}_{4} \mathrm{P}: \mathrm{C}, 51.16 ; \mathrm{H}$, 3.96 ; N, 13.77\%. Found: C, 51.08; H, 3.91; N, 13.70\%.

Dimethyl 1-Benzyl-3-(dicyanomethyl)-2-oxoindolin-3-ylphosphonate $4 \mathrm{~g} 75 \%$ yield. Brown powder. mp $125-130{ }^{\circ} \mathrm{C}$. IR $(\mathrm{KBr})\left(v_{\max } / \mathrm{cm}^{-1}\right)$ : 2255, 1719, 1237. ${ }^{1} \mathrm{H}-\mathrm{NMR}\left(\mathrm{DMSO}-d_{6}\right) \delta: 3.66\left(3 \mathrm{H}, \mathrm{d}, J_{\mathrm{HP}}=11.1 \mathrm{~Hz}\right.$, $\left.\mathrm{OCH}_{3}\right), 3.75\left(3 \mathrm{H}, \mathrm{d}, J_{\mathrm{HP}}=11.1 \mathrm{~Hz}, \mathrm{OCH}_{3}\right), 4.99,5.08\left(2 \mathrm{H}, \mathrm{AB}_{\mathrm{q}}\right.$ $\left.J_{\mathrm{AB}}=15.6 \mathrm{~Hz}, \mathrm{NCH}_{2}\right), 6.16\left(1 \mathrm{H}, \mathrm{d}, J_{\mathrm{HP}}=8.1 \mathrm{~Hz}, \mathrm{CH}(\mathrm{CN})_{2}\right), 7.07-7.63(9 \mathrm{H}$, m, H-Ar). ${ }^{13} \mathrm{C}-\mathrm{NMR}$ (DMSO- $\left.d_{6}\right) \delta: 27.3,52.8\left(\mathrm{~d}, J_{\mathrm{CP}}=138.2 \mathrm{~Hz}, \mathrm{C}-\mathrm{P}\right.$ ), $55.3\left(\mathrm{~d}, J_{\mathrm{CP}}=7.6 \mathrm{~Hz}, \mathrm{OCH}_{3}\right), 56.0\left(\mathrm{~d}, J_{\mathrm{CP}}=6.7 \mathrm{~Hz}, \mathrm{OCH}_{3}\right), 111.0,111.9$, $120.9,124.0,126.1,127.5,128.123,129.0,131.6,135.6,143.6,143.7$, 169.5. ${ }^{31} \mathrm{P}-\mathrm{NMR}$ (DMSO- $d_{6}$ ) $\delta$ : 13.27. Anal. Calcd for $\mathrm{C}_{20} \mathrm{H}_{18} \mathrm{~N}_{3} \mathrm{O}{ }_{4} \mathrm{P}: \mathrm{C}$, $60.76 ; \mathrm{H}, 4.59$; N, 10.63\%. Found: C, 60.85; H, 4.53; N, 10.70\%.

Dimethyl 3-(Dicyanomethyl)-1-methyl-2-oxoindolin-3-ylphosphonate 4h $70 \%$ yield. Cream powder. mp $148-150{ }^{\circ} \mathrm{C}$. IR $(\mathrm{KBr})\left(v_{\max } / \mathrm{cm}^{-1}\right)$ : 2260, 1708, 1266. ${ }^{1} \mathrm{H}-\mathrm{NMR}\left(\mathrm{DMSO}-d_{6}\right) \delta: 3.25\left(3 \mathrm{H}, \mathrm{s}, \mathrm{NCH}_{3}\right), 3.68(3 \mathrm{H}, \mathrm{d}$, $\left.J_{\mathrm{HP}}=11.1 \mathrm{~Hz}, \mathrm{OCH}_{3}\right), 3.75\left(3 \mathrm{H}, \mathrm{d}, J_{\mathrm{HP}}=11.1 \mathrm{~Hz}, \mathrm{OCH}_{3}\right), 6.05(1 \mathrm{H}, \mathrm{d}$, $\left.J_{\mathrm{HP}}=8.7 \mathrm{~Hz}, \mathrm{CH}(\mathrm{CN})_{2}\right), 7.24-7.61(4 \mathrm{H}, \mathrm{m}, \mathrm{H}-\mathrm{Ar}) .{ }^{13} \mathrm{C}-\mathrm{NMR}\left(\mathrm{DMSO}-d_{6}\right)$ $\delta: 27.4,52.8\left(\mathrm{~d}, J_{\mathrm{CP}}=138.8 \mathrm{~Hz}, \mathrm{C}-\mathrm{P}\right), 55.2\left(\mathrm{~d}, J_{\mathrm{CP}}=7.4 \mathrm{~Hz}, \mathrm{OCH}_{3}\right), 56.0(\mathrm{~d}$, $\left.J_{\mathrm{CP}}=6.7 \mathrm{~Hz}, \mathrm{OCH}_{3}\right), 111.4\left(\mathrm{~d}, J_{\mathrm{CP}}=12.4 \mathrm{~Hz}, \mathrm{CN}\right), 111.8\left(\mathrm{~d}, J_{\mathrm{CP}}=8.0 \mathrm{~Hz}\right.$ $\mathrm{CN}), 121.0\left(\mathrm{~d}, J_{\mathrm{CP}}=7.5 \mathrm{~Hz}, \mathrm{C}-\mathrm{Ar}\right), 124.0,125.9$ (d, $\left.J_{\mathrm{CP}}=3.1 \mathrm{~Hz}, \mathrm{C}-\mathrm{Ar}\right)$, 131.6, $144.6\left(\mathrm{~d}, J_{\mathrm{CP}}=6.6 \mathrm{~Hz}, \mathrm{C}-\mathrm{Ar}\right), 169.3\left(\mathrm{~d}, J_{\mathrm{CP}}=3.1 \mathrm{~Hz}, \mathrm{CO}\right) .{ }^{31} \mathrm{P}-\mathrm{NMR}$ (DMSO-d $d_{6} \delta$ : 15.16. Anal. Calcd for $\mathrm{C}_{14} \mathrm{H}_{14} \mathrm{~N}_{3} \mathrm{O}_{4} \mathrm{P}: \mathrm{C}, 52.67 ; \mathrm{H}, 4.42 ; \mathrm{N}$, $13.16 \%$. Found: C, 52.55; H, 4.33; N, 13.09\%.

Dimethyl 3-(Dicyanomethyl)-1-ethyl-2-oxoindolin-3-ylphosphonate 4i $86 \%$ yield. Cream powder. mp $124-126^{\circ} \mathrm{C}$. IR $(\mathrm{KBr})\left(v_{\max } / \mathrm{cm}^{-1}\right): 2254$ 1712, 1277. ${ }^{1} \mathrm{H}-\mathrm{NMR}$ (DMSO- $\left.d_{6}\right) \delta: 1.13\left(3 \mathrm{H}, \mathrm{t}, J_{\mathrm{HH}}=6.6 \mathrm{~Hz}, \mathrm{CH}_{3}\right), 3.65$ $\left(3 \mathrm{H}, \mathrm{d}, J_{\mathrm{HP}}=10.8 \mathrm{~Hz}, \mathrm{OCH}_{3}\right), 3.74\left(3 \mathrm{H}, \mathrm{d}, J_{\mathrm{HP}}=11.1 \mathrm{~Hz}, \mathrm{OCH}_{3}\right), 3.80(2 \mathrm{H}, \mathrm{q}$, $\left.J_{\mathrm{HH}}=7.2 \mathrm{~Hz}, \mathrm{NCH}_{2}\right), 6.03\left(1 \mathrm{H}, \mathrm{d}, J_{\mathrm{HP}}=7.8 \mathrm{~Hz}, \mathrm{CH}(\mathrm{CN})_{2}\right), 7.22-7.61(4 \mathrm{H}$, m, H-Ar). ${ }^{13} \mathrm{C}-\mathrm{NMR}$ (DMSO- $\left.d_{6}\right) \delta: 12.4,27.5,35.5,52.6\left(\mathrm{~d}, J_{\mathrm{CP}}=138.9 \mathrm{~Hz}\right.$, $\mathrm{C}-\mathrm{P}), 55.2\left(\mathrm{~d}, J_{\mathrm{CP}}=7.3 \mathrm{~Hz}, \mathrm{OCH}_{3}\right), 55.9\left(\mathrm{~d}, J_{\mathrm{CP}}=6.7 \mathrm{~Hz}, \mathrm{OCH}_{3}\right), 110.5$, $111.3\left(\mathrm{~d}, J_{\mathrm{CP}}=13.4 \mathrm{~Hz}, \mathrm{CN}\right), 111.8\left(\mathrm{~d}, J_{\mathrm{CP}}=7.6 \mathrm{~Hz}, \mathrm{CN}\right), 121.2,123.8$, $126.2,131.7,143.6$ (d, $J_{\mathrm{CP}}=6.8 \mathrm{~Hz}, \mathrm{C}-\mathrm{Ar}$ ), 169.0. ${ }^{31} \mathrm{P}-\mathrm{NMR}$ (DMSO- $d_{6}$ ) $\delta$ 15.22. Anal. Calcd for $\mathrm{C}_{15} \mathrm{H}_{16} \mathrm{~N}_{3} \mathrm{O}_{4} \mathrm{P}: \mathrm{C}, 54.06 ; \mathrm{H}, 4.84 ; \mathrm{N}, 12.61 \%$. Found: C, $54.16 ; \mathrm{H}, 4.77 ; \mathrm{N}, 12.55 \%$.

Dimethyl 3-(Dicyanomethyl)-5-nitro-2-oxoindolin-3-ylphosphonate $4 \mathbf{j}$ $72 \%$ yield. Orange powder. $\mathrm{mp} 197-199^{\circ} \mathrm{C}$. IR $(\mathrm{KBr})\left(v_{\mathrm{s}} / \mathrm{cm}^{-1}\right): 3111$, 2255, 1747, 1257. ${ }^{1} \mathrm{H}-\mathrm{NMR}\left(\mathrm{DMSO}-d_{6}\right) \delta: 3.72\left(3 \mathrm{H}, \mathrm{d}, J_{\mathrm{HP}}=11.1 \mathrm{~Hz}\right.$, $\left.\mathrm{OCH}_{3}\right), 3.81\left(3 \mathrm{H}, \mathrm{d}, J_{\mathrm{HP}}=11.4 \mathrm{~Hz}, \mathrm{OCH}_{3}\right), 6.25\left(1 \mathrm{H}, \mathrm{d}, J_{\mathrm{HP}}=9.0 \mathrm{~Hz}\right.$ $\left.\mathrm{CH}(\mathrm{CN})_{2}\right), 7.24-8.40$ (3H, m, H-Ar $), 12.14(1 \mathrm{H}, \mathrm{s}, \mathrm{NH}) .{ }^{13} \mathrm{C}-\mathrm{NMR}$ $\left(\mathrm{DMSO}-d_{6}\right) \delta: 26.7,52.9\left(\mathrm{~d}, J_{\mathrm{CP}}=137.4 \mathrm{~Hz}, \mathrm{C}-\mathrm{P}\right), 55.6\left(\mathrm{~d}, J_{\mathrm{CP}}=7.3 \mathrm{~Hz}\right.$ $\left.\mathrm{OCH}_{3}\right), 56.3\left(\mathrm{~d}, J_{\mathrm{CP}}=6.6 \mathrm{~Hz}, \mathrm{OCH}_{3}\right), 111.5,111.7,121.8,122.8,128.6$, $143.1,149.4\left(\mathrm{~d}, J_{\mathrm{CP}}=6.4 \mathrm{~Hz}, \mathrm{C}-\mathrm{Ar}\right), 171.3 .{ }^{31} \mathrm{P}-\mathrm{NMR}$ (DMSO- $\left.d_{6}\right) \delta: 13.81$ Anal. Calcd for $\mathrm{C}_{13} \mathrm{H}_{11} \mathrm{~N}_{4} \mathrm{O}_{6} \mathrm{P}: \mathrm{C}, 44.58 ; \mathrm{H}, 3.17 ; \mathrm{N}, 16.00 \%$. Found: $\mathrm{C}$, 44.51; H, 3.09; N, 15.93\%.

Dimethyl 5-Bromo-3-(dicyanomethyl)-2-oxoindolin-3-ylphosphonate $4 \mathbf{k} 88 \%$ yield. Pink powder. mp $182-186^{\circ} \mathrm{C}$. IR $(\mathrm{KBr})\left(v_{\max } / \mathrm{cm}^{-1}\right)$ : 3432, 2260, 1726, 1251. ${ }^{1} \mathrm{H}-\mathrm{NMR}\left(\mathrm{DMSO}-d_{6}\right) \delta: 3.70\left(3 \mathrm{H}, \mathrm{d}, J_{\mathrm{HP}}=10.8 \mathrm{~Hz}\right.$, $\left.\mathrm{OCH}_{3}\right), 3.78\left(3 \mathrm{H}, \mathrm{d}, J_{\mathrm{HP}}=11.1 \mathrm{~Hz}, \mathrm{OCH}_{3}\right), 6.07\left(1 \mathrm{H}, \mathrm{d}, J_{\mathrm{HP}}=8.4 \mathrm{~Hz}\right.$ $\left.\mathrm{CH}(\mathrm{CN})_{2}\right), 6.98-7.65$ (3H, m, H-Ar $), 11.58(1 \mathrm{H}, \mathrm{s}, \mathrm{NH}) .{ }^{13} \mathrm{C}-\mathrm{NMR}$ $\left(\mathrm{DMSO}-d_{6}\right) \delta: 27.1,53.1\left(\mathrm{~d}, J_{\mathrm{CP}}=138.3 \mathrm{~Hz}, \mathrm{C}-\mathrm{P}\right), 55.3\left(\mathrm{~d}, J_{\mathrm{CP}}=7.5 \mathrm{~Hz}\right.$, $\left.\mathrm{OCH}_{3}\right), 56.1\left(\mathrm{~d}, J_{\mathrm{CP}}=6.8 \mathrm{~Hz}, \mathrm{OCH}_{3}\right), 111.1,111.6,113.3,114.5,124.2(\mathrm{~d}$ $\left.J_{\mathrm{CP}}=5.9 \mathrm{~Hz}, \mathrm{C}-\mathrm{Ar}\right), 128.6,134.4,142.7\left(\mathrm{~d}, J_{\mathrm{CP}}=6.8 \mathrm{~Hz}, \mathrm{C}-\mathrm{Ar}\right), 170.4 .{ }^{31} \mathrm{P}$ NMR (DMSO- $d_{6}$ ) $\delta$ : 14.25. Anal. Calcd for $\mathrm{C}_{13} \mathrm{H}_{11} \mathrm{BrN}_{3} \mathrm{O}_{4} \mathrm{P}: \mathrm{C}, 40.65 ; \mathrm{H}$, 2.89 ; N, 10.94\%. Found: C, 40.53; H, 2.81; N, 10.84\%.
Dimethyl 5-Bromo-3-(dicyanomethyl)-1-methyl-2-oxoindolin-3-ylphosphonate $4 \mathrm{I} \quad 82 \%$ yield. Pink powder. $\mathrm{mp} 168-171^{\circ} \mathrm{C}$. IR $(\mathrm{KBr})\left(v_{\max }\right.$ $\left.\mathrm{cm}^{-1}\right): 2260,1724,1250 .{ }^{1} \mathrm{H}-\mathrm{NMR}\left(\mathrm{DMSO}-d_{6}\right) \delta: 3.25\left(3 \mathrm{H}, \mathrm{s}, \mathrm{CH}_{3}\right), 3.70$ $\left(3 \mathrm{H}, \mathrm{d}, J_{\mathrm{HP}}=10.8 \mathrm{~Hz}, \mathrm{OCH}_{3}\right), 3.79\left(3 \mathrm{H}, \mathrm{d}, J_{\mathrm{HP}}=11.1 \mathrm{~Hz}, \mathrm{OCH}_{3}\right), 7.26(1 \mathrm{H}, \mathrm{d}$, $\left.J_{\mathrm{HP}}=8.4 \mathrm{~Hz}, \mathrm{CH}(\mathrm{CN})_{2}\right), 7.68-7.93(3 \mathrm{H}, \mathrm{m}, \mathrm{H}-\mathrm{Ar}) .{ }^{13} \mathrm{C}-\mathrm{NMR}\left(\mathrm{DMSO}-d_{6}\right)$ $\delta: 27.1,27.6,52.5\left(\mathrm{~d}, J_{\mathrm{CP}}=138.4 \mathrm{~Hz}, \mathrm{C}-\mathrm{P}\right), 55.4\left(\mathrm{~d}, J_{\mathrm{CP}}=7.3 \mathrm{~Hz}, \mathrm{OCH}_{3}\right)$, $55.2\left(\mathrm{~d}, J_{\mathrm{CP}}=6.6 \mathrm{~Hz}, \mathrm{OCH}_{3}\right), 111.5,112.6,115.3,123.2,128.5,134.5,144.0$ $\left(\mathrm{d}, J_{\mathrm{CP}}=6.6 \mathrm{~Hz}, \mathrm{C}-\mathrm{Ar}\right), 168.9 .{ }^{31} \mathrm{P}-\mathrm{NMR}\left(\mathrm{DMSO}-d_{6}\right) \delta$ : 14.48. Anal. Calcd for $\mathrm{C}_{14} \mathrm{H}_{13} \mathrm{BrN}_{3} \mathrm{O}_{4} \mathrm{P}$ : C, 42.23; H, 3.29; N, 10.55\%. Found: C, 42.32; H, 3.23; N, $10.48 \%$.

Diisopropyl 3-(Dicyanomethyl)-2-oxoindolin-3-ylphosphonate $\quad 4 \mathrm{~m}$ $81 \%$ yield. Cream powder. $\mathrm{mp} 199^{\circ} \mathrm{C}$ dec. IR $(\mathrm{KBr})\left(v_{\max } / \mathrm{cm}^{-1}\right): 3185$, 2260, 1723, 1219. ${ }^{1} \mathrm{H}-\mathrm{NMR}$ (DMSO- $\left.d_{6}\right) \delta: 1.03-1.29\left(12 \mathrm{H}, \mathrm{m}, \mathrm{CH}_{3}\right)$, $4.40-4.50(1 \mathrm{H}, \mathrm{m}, \mathrm{OCH}), 4.62-4.70(1 \mathrm{H}, \mathrm{m}, \mathrm{OCH}), 5.73(1 \mathrm{H}, \mathrm{d}$ $\left.J_{\mathrm{HP}}=7.8 \mathrm{~Hz}, \mathrm{CH}(\mathrm{CN})_{2}\right), 6.98-7.59(4 \mathrm{H}, \mathrm{m}, \mathrm{H}-\mathrm{Ar}), 11.32(1 \mathrm{H}, \mathrm{s}, \mathrm{NH}) .{ }^{13} \mathrm{C}-$ NMR (DMSO- $\left.d_{6}\right) \delta: 23.0\left(\mathrm{~d}, J_{\mathrm{CP}}=6.7 \mathrm{~Hz}, \mathrm{CH}_{3}\right), 23.8\left(\mathrm{~d}, J_{\mathrm{CP}}=5.2 \mathrm{~Hz}, \mathrm{CH}_{3}\right)$, $24.0\left(\mathrm{~d}, J_{\mathrm{CP}}=4.0 \mathrm{~Hz}, \mathrm{CH}_{3}\right), 24.4\left(\mathrm{~d}, J_{\mathrm{CP}}=2.3 \mathrm{~Hz}, \mathrm{CH}_{3}\right), 27.8,53.0(\mathrm{~d}$ $\left.J_{\mathrm{CP}}=138.6 \mathrm{~Hz}, \mathrm{C}-\mathrm{P}\right), 73.8\left(\mathrm{~d}, J_{\mathrm{CP}}=7.6 \mathrm{~Hz}, \mathrm{OCH}\right), 74.6\left(\mathrm{~d}, J_{\mathrm{CP}}=7.1 \mathrm{~Hz}\right.$ $\mathrm{OCH}), 111.0,111.7\left(\mathrm{~d}, J_{\mathrm{CP}}=14.4 \mathrm{~Hz}, \mathrm{CN}\right), 112.1\left(\mathrm{~d}, J_{\mathrm{CP}}=7.1 \mathrm{~Hz}, \mathrm{CN}\right), 122.2$ $\left(\mathrm{d}, J_{\mathrm{CP}}=5.4 \mathrm{~Hz}, \mathrm{C}-\mathrm{Ar}\right), 123.0,126.3,131.3,143.5\left(\mathrm{~d}, J_{\mathrm{CP}}=6.8 \mathrm{~Hz}, \mathrm{C}-\mathrm{Ar}\right)$, $171.0\left(\mathrm{~d}, J_{\mathrm{CP}}=3.4 \mathrm{~Hz}, \mathrm{CO}\right) .{ }^{31} \mathrm{P}-\mathrm{NMR}\left(\mathrm{DMSO}-d_{6}\right) \delta: 11.30$. Anal. Calcd for $\mathrm{C}_{17} \mathrm{H}_{20} \mathrm{~N}_{3} \mathrm{O}_{4} \mathrm{P}: \mathrm{C}, 56.51 ; \mathrm{H}, 5.58 ; \mathrm{N}, 11.63 \%$. Found: C, 56.60; H, 5.50; N, $11.74 \%$.

Diisopropyl 1-Benzyl-3-(dicyanomethyl)-2-oxoindolin-3-ylphosphonate 4n $76 \%$ yield. Pale orange powder. mp $117-120^{\circ} \mathrm{C}$. IR $(\mathrm{KBr})$ $\left(v_{\max } / \mathrm{cm}^{-1}\right): 2260,1713,1245 .{ }^{1} \mathrm{H}-\mathrm{NMR}\left(\mathrm{DMSO}-d_{6}\right) \delta: 0.87-1.30(12 \mathrm{H}$ $\left.\mathrm{m}, \mathrm{CH}_{3}\right), 4.42-4.48(1 \mathrm{H}, \mathrm{m}, \mathrm{OCH}), 4.65-4.71(1 \mathrm{H}, \mathrm{m}, \mathrm{OCH}), 4.95-5.06$ $\left(2 \mathrm{H}, \mathrm{m}, \mathrm{NCH}_{2}\right), 5.92\left(1 \mathrm{H}, \mathrm{d}, J_{\mathrm{HP}}=7.8 \mathrm{~Hz}, \mathrm{CH}(\mathrm{CN})_{2}\right), 7.06-7.64(9 \mathrm{H}, \mathrm{m}, \mathrm{H}-$ Ar). ${ }^{13} \mathrm{C}-\mathrm{NMR} \quad\left(\mathrm{DMSO}-d_{6}\right) \quad \delta: 22.8\left(\mathrm{~d}, J_{\mathrm{CP}}=6.1 \mathrm{~Hz}, \mathrm{CH}_{3}\right), 23.9(\mathrm{~d}$, $\left.J_{\mathrm{CP}}=4.3 \mathrm{~Hz}, 2 \mathrm{CH}_{3}\right), 24.4\left(\mathrm{~d}, J_{\mathrm{CP}}=2.2 \mathrm{~Hz}, \mathrm{CH}_{3}\right), 27.6,43.9,52.5(\mathrm{~d}$ $\left.J_{\mathrm{CP}}=138.2 \mathrm{~Hz}, \mathrm{C}-\mathrm{P}\right), 74.1\left(\mathrm{~d}, J_{\mathrm{CP}}=7.9 \mathrm{~Hz}, \mathrm{OCH}\right), 74.9\left(\mathrm{~d}, J_{\mathrm{CP}}=7.5 \mathrm{~Hz}\right.$, $\mathrm{OCH}), 110.8,111.6,121.0,123.8,126.2,127.8,128.1,129.0,131.4,135.7$, 143.8, 143.9, 169.7. ${ }^{31} \mathrm{P}-\mathrm{NMR}$ (DMSO- $\left.d_{6}\right) \delta$ : 11.05. Anal. Calcd for $\mathrm{C}_{24} \mathrm{H}_{26} \mathrm{~N}_{3} \mathrm{O}_{4} \mathrm{P}: \mathrm{C}, 63.85 ; \mathrm{H}, 5.80 ; \mathrm{N}, 9.31 \%$. Found: C, 63.97; H, 5.72; N, $9.37 \%$.

Diisopropyl 3-(Dicyanomethyl)-1-methyl-2-oxoindolin-3-ylphosphonate $4072 \%$ yield. White powder. mp $121-123{ }^{\circ} \mathrm{C}$. IR (KBr) $\left(v_{\max } / \mathrm{cm}^{-1}\right): 2260,1718,1261 .{ }^{1} \mathrm{H}-\mathrm{NMR}\left(\mathrm{DMSO}-d_{6}\right) \delta: 1.01-1.30(12 \mathrm{H}$ $\left.\mathrm{m}, \mathrm{CH}_{3}\right), 3.23\left(3 \mathrm{H}, \mathrm{s}, \mathrm{NCH}_{3}\right), 4.38-4.48(1 \mathrm{H}, \mathrm{m}, \mathrm{OCH}), 4.61-4.71(1 \mathrm{H}$, $\mathrm{m}, \mathrm{OCH}), 5.82\left(1 \mathrm{H}, \mathrm{d}, J_{\mathrm{HP}}=8.1 \mathrm{~Hz}, \mathrm{CH}(\mathrm{CN})_{2}\right), 7.23-7.63(4 \mathrm{H}, \mathrm{m}, \mathrm{H}-\mathrm{Ar})$ ${ }^{13} \mathrm{C}-\mathrm{NMR}$ (DMSO- $d_{6}$ ) $\delta: 23.0\left(\mathrm{~d}, J_{\mathrm{CP}}=6.5 \mathrm{~Hz}, \mathrm{CH}_{3}\right), 23.8\left(\mathrm{~d}, J_{\mathrm{CP}}=5.1 \mathrm{~Hz}\right.$, $\left.\mathrm{CH}_{3}\right), 24.0\left(\mathrm{~d}, J_{\mathrm{CP}}=4.1 \mathrm{~Hz}, \mathrm{CH}_{3}\right), 24.4\left(\mathrm{~d}, J_{\mathrm{CP}}=2.2 \mathrm{~Hz}, \mathrm{CH}_{3}\right), 27.3,27.7,52.5$ $\left(\mathrm{d}, J_{\mathrm{CP}}=138.6 \mathrm{~Hz}, \mathrm{C}-\mathrm{P}\right), 74.0\left(\mathrm{~d}, J_{\mathrm{CP}}=7.6 \mathrm{~Hz}, \mathrm{OCH}\right), 74.7$ (d, $J_{\mathrm{CP}}=7.0 \mathrm{~Hz}$, $\mathrm{OCH}), 110.2,111.6\left(\mathrm{~d}, J_{\mathrm{CP}}=13.4 \mathrm{~Hz}, \mathrm{CN}\right), 112.0,121.3\left(\mathrm{~d}, J_{\mathrm{CP}}=5.4 \mathrm{~Hz}, \mathrm{C}\right.$ Ar), 123.7, 126.1, 131.5, $144.7\left(\mathrm{~d}, J_{\mathrm{CP}}=6.7 \mathrm{~Hz}, \mathrm{C}-\mathrm{Ar}\right), 169.4 .{ }^{31} \mathrm{P}-\mathrm{NMR}$ $\left(\mathrm{DMSO}-d_{6}\right) \delta$ : 11.07. Anal. Calcd for $\mathrm{C}_{18} \mathrm{H}_{22} \mathrm{~N}_{3} \mathrm{O}_{4} \mathrm{P}: \mathrm{C}, 57.60 ; \mathrm{H}, 5.91 ; \mathrm{N}$, $11.19 \%$. Found: C, 57.51; H, 5.84; N, 11.11\%.

Diisopropyl 3-(Dicyanomethyl)-1-ethyl-2-oxoindolin-3-ylphosphonate 4 p $74 \%$ yield. White powder. mp $108-112^{\circ} \mathrm{C}$. IR $(\mathrm{KBr})\left(v_{\max } / \mathrm{cm}^{-1}\right)$ : $3416,2260,1708,1245$. ${ }^{1} \mathrm{H}-\mathrm{NMR}\left(\mathrm{DMSO}-d_{6}\right) \delta: 0.97-0.98(3 \mathrm{H}$, brs, $\left.\mathrm{CH}_{3}\right), 1.19-1.29\left(12 \mathrm{H}, \mathrm{m}, \mathrm{CH}_{3}\right), 3.78-3.80\left(2 \mathrm{H}, \mathrm{m}, \mathrm{NCH}_{2}\right), 4.41-4.45$ $(1 \mathrm{H}, \mathrm{m}, \mathrm{OCH}), 4.63-4.69(1 \mathrm{H}, \mathrm{m}, \mathrm{OCH}), 5.79\left(1 \mathrm{H}, \mathrm{d}, J_{\mathrm{HP}}=7.5 \mathrm{~Hz}\right.$ $\left.\mathrm{CH}(\mathrm{CN})_{2}\right), 7.21-7.63$ (4H, m, H-Ar). ${ }^{13} \mathrm{C}-\mathrm{NMR}$ (DMSO- $\left.d_{6}\right) \delta: 12.5,23.0$ $\left(\mathrm{d}, J_{\mathrm{CP}}=6.4 \mathrm{~Hz}, \mathrm{CH}_{3}\right), 23.8\left(\mathrm{~d}, J_{\mathrm{CP}}=6.2 \mathrm{~Hz}, \mathrm{CH}_{3}\right), 23.9\left(\mathrm{~d}, J_{\mathrm{CP}}=4.5 \mathrm{~Hz}, \mathrm{CH}_{3}\right)$, $24.4\left(\mathrm{~d}, J_{\mathrm{CP}}=2.7 \mathrm{~Hz}, \mathrm{CH}_{3}\right), 27.8,35.4,52.3\left(\mathrm{~d}, J_{\mathrm{CP}}=139.0 \mathrm{~Hz}, \mathrm{C}-\mathrm{P}\right), 74.0(\mathrm{~d}$, $\left.J_{\mathrm{CP}}=7.8 \mathrm{~Hz}, \mathrm{OCH}\right), 74.7\left(\mathrm{~d}, J_{\mathrm{CP}}=6.7 \mathrm{~Hz}, \mathrm{OCH}\right), 110.3,111.4,111.6,121.5$, 123.5, 126.3, 131.5, 143.7 (d, $J_{\mathrm{CP}}=6.7 \mathrm{~Hz}, \mathrm{C}-\mathrm{Ar}$ ), 169.1. ${ }^{31} \mathrm{P}-\mathrm{NMR}$ (DMSO$\left.d_{6}\right) \delta$ : 11.15. Anal. Calcd for $\mathrm{C}_{19} \mathrm{H}_{24} \mathrm{~N}_{3} \mathrm{O}_{4} \mathrm{P}: \mathrm{C}, 58.61 ; \mathrm{H}, 6.21 ; \mathrm{N}, 10.79 \%$. Found: C, 58.50; H, 6.29; N, 10.90\%.

Diisopropyl 3-(Dicyanomethyl)-5-nitro-2-oxoindolin-3-ylphosphonate $4 q 71 \%$ yield. Yellow powder. $\mathrm{mp} 175^{\circ} \mathrm{C}$ dec. IR $(\mathrm{KBr})\left(v_{\max } / \mathrm{cm}^{-1}\right)$ : 3190, 2265, 1729, 1261. ${ }^{1} \mathrm{H}-\mathrm{NMR}$ (DMSO-d $) \delta: 1.12-1.31(12 \mathrm{H}, \mathrm{m}$, $\left.\mathrm{CH}_{3}\right), 4.52-4.58(1 \mathrm{H}, \mathrm{m}, \mathrm{OCH}), 4.66-4.73(1 \mathrm{H}, \mathrm{m}, \mathrm{OCH}), 6.00(1 \mathrm{H}, \mathrm{d}$, $\left.J_{\mathrm{HP}}=8.4 \mathrm{~Hz}, \mathrm{CH}(\mathrm{CN})_{2}\right), 7.23-8.40(3 \mathrm{H}, \mathrm{m}, \mathrm{H}-\mathrm{Ar}), 12.10(1 \mathrm{H}, \mathrm{s}, \mathrm{NH}) .{ }^{13} \mathrm{C}-$ NMR (DMSO- $\left.d_{6}\right) \delta: 23.1\left(\mathrm{~d}, J_{\mathrm{CP}}=6.6 \mathrm{~Hz}, \mathrm{CH}_{3}\right), 23.6\left(\mathrm{~d}, J_{\mathrm{CP}}=5.4 \mathrm{~Hz}, \mathrm{CH}_{3}\right)$, $24.0\left(\mathrm{~d}, J_{\mathrm{CP}}=3.7 \mathrm{~Hz}, \mathrm{CH}_{3}\right), 24.4\left(\mathrm{~d}, J_{\mathrm{CP}}=1.7 \mathrm{~Hz}, \mathrm{CH}_{3}\right), 27.2,53.0(\mathrm{~d}$ $\left.J_{\mathrm{CP}}=137.2 \mathrm{~Hz}, \mathrm{C}-\mathrm{P}\right), 74.7\left(\mathrm{~d}, J_{\mathrm{CP}}=7.9 \mathrm{~Hz}, \mathrm{OCH}\right), 75.4\left(\mathrm{~d}, J_{\mathrm{CP}}=6.6 \mathrm{~Hz}\right.$, $\mathrm{OCH}), 111.5,122.0,123.2,123.3,128.4,142.9$ (d, $\left.J_{\mathrm{CP}}=3.1 \mathrm{C}-\mathrm{Ar}\right), 149.6$, 149.7, 171.4. ${ }^{31} \mathrm{P}-\mathrm{NMR}$ (DMSO- $d_{6}$ ) $\delta$ : 9.78. Anal. Calcd for $\mathrm{C}_{17} \mathrm{H}_{19} \mathrm{~N}_{4} \mathrm{O}_{6} \mathrm{P}$ : C, $50.25 ; \mathrm{H}, 4.71$; N, $13.79 \%$. Found: C, 50.19; H, 4.76; N, 13.72\%.

Diisopropyl 5-Bromo-3-(dicyanomethyl)-2-oxoindolin-3-ylphosphonate $4 \mathbf{r} 79 \%$ yield. White powder. $\operatorname{mp~} 175^{\circ} \mathrm{C}$ dec. IR $(\mathrm{KBr})\left(v_{\max } / \mathrm{cm}^{-1}\right)$ : 3437, 2260, 1734, 1240. ${ }^{1} \mathrm{H}-\mathrm{NMR}$ (DMSO- $\left.d_{6}\right) \delta: 1.09-1.31(12 \mathrm{H}, \mathrm{m}$, $\left.\mathrm{CH}_{3}\right), 4.46-4.54(1 \mathrm{H}, \mathrm{m}, \mathrm{OCH}), 4.56-4.73(1 \mathrm{H}, \mathrm{m}, \mathrm{OCH}), 4.56-5.06$ 
$\left(2 \mathrm{H}, \mathrm{m}, \mathrm{NCH}_{2}\right), 5.83\left(1 \mathrm{H}, \mathrm{d}, J_{\mathrm{HP}}=7.8 \mathrm{~Hz}, \mathrm{CH}(\mathrm{CN})_{2}\right), 6.98-7.64(3 \mathrm{H}, \mathrm{m}, \mathrm{H}-$ Ar), $11.51(1 \mathrm{H}, \mathrm{s}, \mathrm{NH}) .{ }^{13} \mathrm{C}-\mathrm{NMR}\left(\mathrm{DMSO}-d_{6}\right) \delta: 23.1\left(\mathrm{~d}, J_{\mathrm{CP}}=6.0 \mathrm{~Hz}, \mathrm{CH}_{3}\right)$ $23.7\left(\mathrm{~d}, J_{\mathrm{CP}}=5.1 \mathrm{~Hz}, \mathrm{CH}_{3}\right), 24.0\left(\mathrm{~d}, J_{\mathrm{CP}}=3.8 \mathrm{~Hz}, \mathrm{CH}_{3}\right), 24.4\left(\mathrm{~d}, J_{\mathrm{CP}}=1.7 \mathrm{~Hz}\right.$, $\left.\mathrm{CH}_{3}\right), 27.4,53.0\left(\mathrm{~d}, J_{\mathrm{CP}}=137.5 \mathrm{~Hz}, \mathrm{C}-\mathrm{P}\right), 74.3\left(\mathrm{~d}, J_{\mathrm{CP}}=7.3 \mathrm{~Hz}, \mathrm{OCH}\right), 75.0$ $\left(\mathrm{d}, J_{\mathrm{CP}}=6.7 \mathrm{~Hz}, \mathrm{OCH}\right), 111.5,111.8,113.1,114.2,124.5,128.9,134.1$, $142.8\left(\mathrm{~d}, J_{\mathrm{CP}}=6.5 \mathrm{~Hz}, \mathrm{H}-\mathrm{Ar}\right), 170.6 .{ }^{31} \mathrm{P}-\mathrm{NMR}\left(\mathrm{DMSO}-d_{6}\right) \delta$ : 10.57. Anal. Calcd for $\mathrm{C}_{17} \mathrm{H}_{19} \mathrm{BrN}_{3} \mathrm{O}_{4} \mathrm{P}: \mathrm{C}, 46.38 ; \mathrm{H}, 4.35 ; \mathrm{N}, 9.55 \%$. Found: $\mathrm{C}, 46.30 ; \mathrm{H}$, $4.28 ; \mathrm{N}, 9.46 \%$

Diisopropyl 5-Bromo-3-(dicyanomethyl)-1-methyl-2-oxoindolin-3-ylphosphonate $4 \mathrm{~s} \quad 80 \%$ yield. Cream powder. mp $110-112^{\circ} \mathrm{C}$. IR $(\mathrm{KBr})$ $\left(v_{\max } / \mathrm{cm}^{-1}\right): 2260,1728,1261 .{ }^{1} \mathrm{H}-\mathrm{NMR}\left(\mathrm{DMSO}-d_{6}\right) \delta: 0.84-1.30(12 \mathrm{H}$, $\left.\mathrm{m}, \mathrm{CH}_{3}\right), 3.35\left(3 \mathrm{H}, \mathrm{s}, \mathrm{CH}_{3}\right), 4.43-4.53(1 \mathrm{H}, \mathrm{m}, \mathrm{OCH}), 4.62-4.72(1 \mathrm{H}, \mathrm{m}$ $\mathrm{OCH}), 5.91\left(1 \mathrm{H}, \mathrm{d}, J_{\mathrm{HP}}=8.4 \mathrm{~Hz}, \mathrm{CH}(\mathrm{CN})_{2}\right), 7.23-7.77(3 \mathrm{H}, \mathrm{m}, \mathrm{H}-\mathrm{Ar}) .{ }^{13} \mathrm{C}-$ NMR (DMSO- $\left.d_{6}\right) \delta: 23.1\left(\mathrm{~d}, J_{\mathrm{CP}}=6.4 \mathrm{~Hz}, \mathrm{CH}_{3}\right), 23.7\left(\mathrm{~d}, J_{\mathrm{CP}}=5.2 \mathrm{~Hz}, \mathrm{CH}_{3}\right.$ ), $23.9\left(\mathrm{~d}, J_{\mathrm{CP}}=4.1 \mathrm{~Hz}, \mathrm{CH}_{3}\right), 24.3\left(\mathrm{~d}, J_{\mathrm{CP}}=2.7 \mathrm{~Hz}, \mathrm{CH}_{3}\right), 27.4,27.5,52.4(\mathrm{~d}$ $\left.J_{\mathrm{CP}}=137.9 \mathrm{~Hz}, \mathrm{C}-\mathrm{P}\right), 74.4\left(\mathrm{~d}, J_{\mathrm{CP}}=7.6 \mathrm{~Hz}, \mathrm{OCH}\right), 75.1\left(\mathrm{~d}, J_{\mathrm{CP}}=7.2 \mathrm{~Hz}\right.$, $\mathrm{OCH}), 111.4,111.7,112.4,115.0,123.7\left(\mathrm{~d}, J_{\mathrm{CP}}=5.9 \mathrm{~Hz}, \mathrm{C}-\mathrm{Ar}\right), 128.7$, 134.2, 135.7, 144.1 (d, $\left.J_{\mathrm{CP}}=6.2 \mathrm{~Hz}, \mathrm{C}-\mathrm{Ar}\right), 169.0 .{ }^{31} \mathrm{P}-\mathrm{NMR}$ (DMSO- $d_{6}$ ) $\delta$ 10.35. Anal. Calcd for $\mathrm{C}_{18} \mathrm{H}_{21} \mathrm{BrN}_{3} \mathrm{O}_{4} \mathrm{P}: \mathrm{C}, 47.59 ; \mathrm{H}, 4.66 ; \mathrm{N}, 9.25 \%$. Found: C, $47.47 ; \mathrm{H}, 4.57 ; \mathrm{N}, 9.15 \%$.

Diisopropyl 3-(Dicyanomethyl)-1-methyl-5-nitro-2-oxoindolin-3-ylphosphonate $4 \mathrm{t} \quad 81 \%$ yield. Yellow powder. mp $136-138^{\circ} \mathrm{C}$. IR $(\mathrm{KBr})$ $\left(v_{\max } / \mathrm{cm}^{-1}\right): 2255,1738,1256 .{ }^{1} \mathrm{H}-\mathrm{NMR}$ (DMSO- $\left.d_{6}\right) \delta: 1.03-1.38(12 \mathrm{H}$, $\left.\mathrm{m}, \mathrm{CH}_{3}\right), 3.32(3 \mathrm{H}, \mathrm{s}, \mathrm{CH} 3), 4.46-4.54(1 \mathrm{H}, \mathrm{m}, \mathrm{OCH}), 4.51-4.57(1 \mathrm{H}, \mathrm{m}$ $\mathrm{OCH}), 4.67-4.73\left(2 \mathrm{H}, \mathrm{m}, \mathrm{NCH}_{2}\right), 6.06\left(1 \mathrm{H}, \mathrm{d}, J_{\mathrm{HP}}=9.3 \mathrm{~Hz}, \mathrm{CH}(\mathrm{CN})_{2}\right)$, $7.51-8.53(3 \mathrm{H}, \mathrm{m}, \mathrm{H}-\mathrm{Ar}), 11.51(1 \mathrm{H}, \mathrm{s}, \mathrm{NH}) .{ }^{13} \mathrm{C}-\mathrm{NMR}\left(\mathrm{DMSO}-d_{6}\right) \delta$ $23.1\left(\mathrm{~d}, J_{\mathrm{CP}}=6.4 \mathrm{~Hz}, \mathrm{CH}_{3}\right), 23.7\left(\mathrm{~d}, J_{\mathrm{CP}}=5.3 \mathrm{~Hz}, \mathrm{CH}_{3}\right), 24.0\left(\mathrm{~d}, J_{\mathrm{CP}}=3.7 \mathrm{~Hz}\right.$, $\left.\mathrm{CH}_{3}\right), 24.3\left(\mathrm{~d}, J_{\mathrm{CP}}=2.2 \mathrm{~Hz}, \mathrm{CH}_{3}\right), 27.1,27.9,52.2\left(\mathrm{~d}, J_{\mathrm{CP}}=137.2 \mathrm{~Hz}, \mathrm{C}-\mathrm{P}\right)$ $74.9\left(\mathrm{~d}, J_{\mathrm{CP}}=7.6 \mathrm{~Hz}, \mathrm{OCH}\right), 75.2\left(\mathrm{~d}, J_{\mathrm{CP}}=7.2 \mathrm{~Hz}, \mathrm{OCH}\right), 110.9,111.4,121.6$, $122.3,128.4,143.5,150.2,150.3,171.2 .{ }^{31} \mathrm{P}-\mathrm{NMR}$ (DMSO- $d_{6}$ ) $\delta: 9.62$ Anal. Calcd for $\mathrm{C}_{18} \mathrm{H}_{21} \mathrm{~N}_{4} \mathrm{O}_{6} \mathrm{P}: \mathrm{C}, 51.43 ; \mathrm{H}, 5.04 ; \mathrm{N}, 13.33 \%$. Found: $\mathrm{C}$, $51.52 ; \mathrm{H}, 5.01 ; \mathrm{N}, 13.25 \%$.

Diethyl 1-(Dicyanomethyl)-2-oxo-1,2-dihydroacenaphthylen-1-ylphosphonate 7a $89 \%$ yield. Green powder. mp $158-162{ }^{\circ} \mathrm{C}$. IR $(\mathrm{KBr})$ $\left(v_{\max } / \mathrm{cm}^{-1}\right): 2249,2223,1723,1240 .{ }^{1} \mathrm{H}-\mathrm{NMR}\left(\mathrm{DMSO}-d_{6}\right) \delta: 0.095(3 \mathrm{H}, \mathrm{t}$, $\left.J_{\mathrm{HH}}=6.1 \mathrm{~Hz} \mathrm{CH}_{3}\right), 1.18\left(3 \mathrm{H}, \mathrm{t}, J_{\mathrm{HH}}=7.0 \mathrm{~Hz}, \mathrm{CH}_{3}\right), 3.83-4.11\left(4 \mathrm{H}, \mathrm{m}, \mathrm{CH}_{2}\right)$ $6.15\left(1 \mathrm{H}, \mathrm{d}, J_{\mathrm{HP}}=8.6 \mathrm{~Hz}, \mathrm{CH}(\mathrm{CN})_{2}\right), 7.87-8.48(6 \mathrm{H}, \mathrm{m}, \mathrm{H}-\mathrm{Ar}) .{ }^{13} \mathrm{C}-\mathrm{NMR}$ $\left(\right.$ DMSO- $\left.d_{6}\right) \delta: 16.2\left(\mathrm{~d}, J_{\mathrm{CP}}=5.5 \mathrm{~Hz}, \mathrm{CH}_{3}\right), 16.4\left(\mathrm{~d}, J_{\mathrm{CP}}=5.5 \mathrm{~Hz}, \mathrm{CH}_{3}\right), 27.0$ $57.4\left(\mathrm{~d}, J_{\mathrm{CP}}=135.8 \mathrm{~Hz}, \mathrm{C}-\mathrm{P}\right), 64.7\left(\mathrm{~d}, J_{\mathrm{CP}}=7.4 \mathrm{~Hz}, \mathrm{OCH}_{2}\right), 65.2(\mathrm{~d}$, $\left.J_{\mathrm{CP}}=6.7 \mathrm{~Hz}, \mathrm{OCH}_{2}\right), 111.9,112.3,124.4,124.6,127.7,129.5,129.8,130.9$, 134.0, 134.2, 142.2, 195.1. ${ }^{31} \mathrm{P}-\mathrm{NMR}$ (DMSO- $d_{6}$ ) $\delta$ : 13.47. Anal. Calcd for $\mathrm{C}_{19} \mathrm{H}_{17} \mathrm{~N}_{2} \mathrm{O}_{4} \mathrm{P}: \mathrm{C}, 61.96 ; \mathrm{H}, 4.65 ; \mathrm{N}, 7.61 \%$. Found: $\mathrm{C}, 61.89 ; \mathrm{H}, 4.60 ; \mathrm{N}$ $7.55 \%$.

Dimethyl 1-(Dicyanomethyl)-2-oxo-1,2-dihydroacenaphthylen-1-ylphosphonate $7 \mathbf{b} \quad 80 \%$ yield. Green powder. $\mathrm{mp} 125-130{ }^{\circ} \mathrm{C}$. IR $(\mathrm{KBr})$ $\left(v_{\mathrm{max}} / \mathrm{cm}^{-1}\right): 2234,1718,1251 .{ }^{1} \mathrm{H}-\mathrm{NMR}$ (DMSO- $\left.d_{6}\right) \delta: 0.62-1.25(12 \mathrm{H}$ $\left.\mathrm{m}, \mathrm{CH}_{3}\right), 3.78-3.80\left(2 \mathrm{H}, \mathrm{m}, \mathrm{NCH}_{2}\right), 4.22-4.32(1 \mathrm{H}, \mathrm{m}, \mathrm{OCH}), 4.58-4.68$ $(1 \mathrm{H}, \mathrm{m}, \mathrm{OCH}), 6.02\left(1 \mathrm{H}, \mathrm{d}, J_{\mathrm{HP}}=8.1 \mathrm{~Hz}, \mathrm{CH}(\mathrm{CN})_{2}\right), 7.87-8.47(6 \mathrm{H}, \mathrm{m}, \mathrm{H}-$ Ar). ${ }^{13} \mathrm{C}-\mathrm{NMR}$ (DMSO- $\left.d_{6}\right) \delta: 27.0,55.1\left(\mathrm{~d}, J_{\mathrm{CP}}=7.3 \mathrm{~Hz}, \mathrm{OCH}_{3}\right), 55.6(\mathrm{~d}$, $\left.J_{\mathrm{CP}}=6.7 \mathrm{~Hz}, \mathrm{OCH}_{3}\right), 57.5\left(\mathrm{~d}, J_{\mathrm{CP}}=136.8 \mathrm{~Hz}, \mathrm{C}-\mathrm{P}\right), 111.7\left(\mathrm{~d}, J_{\mathrm{CP}}=13.7 \mathrm{~Hz}\right.$, $\mathrm{CN}), 112.2\left(\mathrm{~d}, J_{\mathrm{CP}}=7.6 \mathrm{~Hz}, \mathrm{CN}\right), 124.5\left(\mathrm{~d}, J_{\mathrm{CP}}=2.9 \mathrm{~Hz}, \mathrm{C}-\mathrm{Ar}\right), 124.8,127.8$, $129.5,129.7,129.9,130.7\left(\mathrm{~d}, J_{\mathrm{CP}}=5.0 \mathrm{~Hz}, \mathrm{C}-\mathrm{Ar}\right), 130.9,134.3,142.2(\mathrm{~d}$, $\left.J_{\mathrm{CP}}=6.1 \mathrm{~Hz}, \mathrm{C}-\mathrm{Ar}\right), 195.0\left(\mathrm{~d}, J_{\mathrm{CP}}=3.5 \mathrm{~Hz}, \mathrm{CO}\right) .{ }^{31} \mathrm{P}-\mathrm{NMR}\left(\mathrm{DMSO}-d_{6}\right) \delta$ : 15.98. Anal. Calcd for $\mathrm{C}_{17} \mathrm{H}_{13} \mathrm{~N}_{2} \mathrm{O}_{4} \mathrm{P}: \mathrm{C}, 60.01 ; \mathrm{H}, 3.85 ; \mathrm{N}, 8.23 \%$. Found: $\mathrm{C}$, 59.92; H, 3.78; N, 8.14\%

Diisopropyl 1-(Dicyanomethyl)-2-oxo-1,2-dihydroacenaphthylen-1-ylphosphonat $7 \mathrm{c} 92 \%$ yield. Green powder. mp $160-162^{\circ} \mathrm{C}$. IR $(\mathrm{KBr})$ $\left(v_{\mathrm{max}} / \mathrm{cm}^{-1}\right): 2254,2228,1734,1229 .{ }^{1} \mathrm{H}-\mathrm{NMR}$ (DMSO- $\left.d_{6}\right) \delta: 0.62-1.25$ $\left(12 \mathrm{H}, \mathrm{m}, \mathrm{CH}_{3}\right), 3.78-3.80\left(2 \mathrm{H}, \mathrm{m}, \mathrm{NCH}_{2}\right), 4.22-4.32(1 \mathrm{H}, \mathrm{m}, \mathrm{OCH})$, $4.58-4.68(1 \mathrm{H}, \mathrm{m}, \mathrm{OCH}), 6.02\left(1 \mathrm{H}, \mathrm{d}, J_{\mathrm{HP}}=8.1 \mathrm{~Hz}, \mathrm{CH}(\mathrm{CN})_{2}\right), 7.87-8.47$ $(6 \mathrm{H}, \mathrm{m}, \mathrm{H}-\mathrm{Ar}) .{ }^{13} \mathrm{C}-\mathrm{NMR}\left(\mathrm{DMSO}-d_{6}\right) \delta: 22.6\left(\mathrm{~d}, J_{\mathrm{CP}}=6.7 \mathrm{~Hz}, \mathrm{CH}_{3}\right), 23.8(\mathrm{~d}$, $\left.J_{\mathrm{CP}}=5.2 \mathrm{~Hz}, \mathrm{CH}_{3}\right), 23.9\left(\mathrm{~d}, J_{\mathrm{CP}}=3.8 \mathrm{~Hz}, \mathrm{CH}_{3}\right), 24.3\left(\mathrm{~d}, J_{\mathrm{CP}}=2.4 \mathrm{~Hz}, \mathrm{CH}_{3}\right)$ $27.2,57.4\left(\mathrm{~d}, J_{\mathrm{CP}}=136.4 \mathrm{~Hz}, \mathrm{C}-\mathrm{P}\right), 73.9\left(\mathrm{~d}, J_{\mathrm{CP}}=7.1 \mathrm{~Hz}, \mathrm{OCH}\right), 74.5(\mathrm{~d}$, $\left.J_{\mathrm{CP}}=7.1 \mathrm{~Hz}, \mathrm{OCH}\right), 111.9,112.0,112.3,112.4,124.4,127.6,129.4,129.8$ $130.8,131.0,134.1,142.2$ (d, $J_{\mathrm{CP}}=5.8 \mathrm{~Hz}, \mathrm{C}-\mathrm{Ar}$ ), 195.0. ${ }^{31} \mathrm{P}-\mathrm{NMR}$ (DMSO$\left.d_{6}\right) \delta$ : 11.67. Anal. Calcd for $\mathrm{C}_{21} \mathrm{H}_{21} \mathrm{~N}_{2} \mathrm{O}_{4} \mathrm{P}: \mathrm{C}, 63.63 ; \mathrm{H}, 5.34 ; \mathrm{N}, 7.07 \%$. Found: C, 63.70; H, 5.39; N, 7.01\%.

Acknowledgements We gratefully acknowledge financial support from the Research Council of Shahid Beheshti University.

\footnotetext{
References

1) Bienayme H., Hulme C., Oddon G., Schmitt P., Chem. Eur. J., 6 , $3321-3329(2000)$.
}

2) Tietze L. F., Modi A., Med. Res. Rev., 20, 304-322 (2000)

3) Dömling A., Ugi I., Angew. Chem. Int. Ed., 39, 3169-3210 (2000).

4) Zhu J., Eur. J. Org. Chem., 2003, 1133-1144 (2003).

5) Nair V., Vinod A. U., Rajesh C., J. Org. Chem., 66, 4427-4429 (2001).

6) List B., Castello C., Synlett, 2001, 1687-1689 (2001)

7) Shestopalov A. M., Emeliyanova Y. M., Shestiopolov A. A., Rodinovskaya L. A., Niazimbetova Z. I., Evans D. H., Org. Lett., 4, 423425 (2002).

8) Bertozzi F., Gustafsson M., Olsson R., Org. Lett., 4, 3147-3150 (2002).

9) Yuan Y., Li X., Ding K. Org. Lett., 4, 3309-3311 (2002).

10) Joshi K. C., Pathak V. N., Jain S. K., Pharmazie, 35, 677-679 (1980).

11) Bolotov V. V., Drugovina V. V., Yakovleva L. V., Bereznyakova A. I., Khim. Farm. Zh., 16, 58 - 61 (1982).

12) Pajouhesh H., Parsons R., Popp F. D., J. Pharm. Sci., 72, 318-321 (1983).

13) Popp F. D., J. Heterocycl. Chem., 21, 1367-1368 (1984).

14) Garrido F., Ibannez J., Gonalons E., Giraldez A., Eur. J. Med. Chem., 10, 143-146 (1975).

15) Kamano Y., Zhang H. P., Ichihara Y., Kizu H., Komiyama K., Itokawa H., Pettit G. R., Tetrahedron Lett., 36, 2783-2784 (1995).

16) Klumpp D. A., Yeung K. Y., Prakash G. K. S., Olah G. A., J. Org. Chem., 63, 4481-4484 (1998).

17) Jursic B., Stevens E. D., Tetrahedron Lett., 43, 5681—5683 (2002).

18) Azizian J., Mohammadi A. A., Karimi N., Mohammadizadeh M. R., Karimi A. R., Catal. Commun., 7, 752-755 (2006).

19) Wang S. Y., Ji S. J., Tetrahedron, 62, 1527-1535 (2006)

20) Yadav J. S., Reddy B. V. S., Gayathri K. U., Meraj S., Prasad A. R., Synthesis, 2006, 4121-4123 (2006)

21) Arbusov B. A., Pure Appl. Chem., 9, 307-335 (1964)

22) Horiguchi M., Kandatsu M., Nature (London), 184, 901—902 (1959).

23) Hildebrand R., "The Role of Phosphonates in Living Systems," CRC, Boca Raton, FL, 1983.

24) Engel R., Chem. Rev., 77, 349-367 (1977)

25) Schug K. A., Lindner W., Chem. Rev., 105, 67-113 (2005).

26) Palacios F., Alonso C., de los Santos J. M., Curr. Org. Chem., 8, $1481-1496$ (2004)

27) Dingwall J. G., Campell C. D., Baylis E. K., U.K. Patent 1542938 (1979) [Chem. Abstr., 88, 105559j (1979)].

28) Kafarski P., Lejczak B., Tyka R., Koba L., Pliszczak E., Wieczorek P. J. Plant Growth Regul., 14, 199-203 (1995).

29) Ishiguri Y., Yamada Y., Kato T., Sasaki M., Mukai K., Eur. Patent EP 82-301905 (1982) [Chem. Abstr., 98, $102686 u$ (1983)].

30) Fields S. C., Tetrahedron, 55, 12237-12273 (1999).

31) Seto, H., Kuzuyama T., Nat. Prod. Rep., 16, 589-596 (1999).

32) Pudovik A. N., Konovalova I. V., Synthesis, 1979, 81-69 (1979).

33) Enders D., Saint-Dizier A., Lannou M. I., Lenzen A., Eur. J. Org. Chem., 2006, 29-49 (2006)

34) Bodalski R., Pietrusiewicz K., Tetrahedron Lett., 13, 4209-4212 (1972).

35) Simoni D., Invidiata F. P., Manferdini M., Lampronti I., Rondanin R., Roberti M., Pollini G. P., Tetrahedron Lett., 39, 7615-7618 (1998).

36) Green K., Tetrahedron Lett., 30, 4807-4810 (1989).

37) Hosseini-Sarvari M., Etemad S., Tetrahedron, 64, 5519-5523 (2008).

38) Stockland R. A. Jr., Taylor R. I., Thompson L. E., Patel P. B., Org Lett., 7, 851-853 (2005).

39) Han L.-B., Zhao C.-Q., J. Org. Chem., 70, 10121-10123 (2005).

40) Xu Q., Han L.-B., Org. Lett., 10, 2099-2101 (2006).

41) Shulyupin M. O., Kazankova M. A., Beletskaya I. P., Org. Lett., 4, $761-763$ (2002).

42) Khidre M. D., Phosphorus, Sulfur and Silicon, 178, 2147-2158 (2003).

43) Yadav J. S., Reddy B. V. S., Sreedhar P., Adv. Synth. Catal., 345, 564 567 (2003)

44) Bazgir A., Seyyedhamzeh M., Yasaei Z., Mirzaei P., Tetrahedron Lett., 48, 8790-8794 (2007)

45) Sayyafi M., Seyyedhamzeh M., Khavasi H. R., Bazgir A., Tetrahedron, 64, 2375-2378 (2008)

46) Dabiri M., Arvin-Nezhad H., Khavasi H. R., Bazgir A., J. Heterocycl. Chem., 44, 1009-1011 (2007).

47) Dabiri M., Azimi S. C., Arvin-Nezhad H., Bazgir A., Heterocycles, 75 87-93 (2008)

48) Dabiri M., Delbari A. S., Bazgir A., Synlett, 2007, 821—823 (2007). 
49) Dabiri M., Arvin-Nezhad H., Khavasi H. R., Bazgir A., Tetrahedron, 63, 1770-1774 (2007).

50) Dabiri M., Delbari A. S., Bazgir A., Heterocycles, 71, 543-548 (2007).

51) Ghahremanzadeh R., Imani Shakibaei G., Bazgir A., Synlett, 2008, 1129-1132 (2008).

52) Imani Shakibaei G., Khavasi H. R., Mirzaei P., Bazgir A., J. Heterocycl. Chem., 45, 1481-1484 (2008).

53) Bazgir A., Noroozi Tisseh Z., Mirzaei P., Tetrahedron Lett., 49, 5165-5168 (2008).
54) Jadidi K., Ghahremanzadeh R., Bazgir A., Tetrahedron, 65, 20052009 (2009).

55) Dabiri M., Azimi S. C., Khavasi H. R., Bazgir A., Tetrahedron, 64, 7307-7311 (2008)

56) Ahadi S., Khavasi H. R., Bazgir A., Chem. Pharm. Bull., 56, 1328 1330 (2008).

57) Ghahremanzadeh R., Sayyafi M., Ahadi S., Bazgir A., J. Comb. Chem., 11, 393-396 (2009).

58) Jadidi K., Ghahremanzadeh R., Bazgir A., J. Comb. Chem., 11, 341344 (2009). 